\title{
Flight Test Maneuvers for Efficient Aerodynamic Modeling
}

\author{
Eugene A. Morelli ${ }^{1}$ \\ NASA Langley Research Center, Hampton, Virginia, 23681
}

\begin{abstract}
Novel flight test maneuvers for efficient aerodynamic modeling were developed and demonstrated in flight. Orthogonal optimized multi-sine inputs were applied to aircraft control surfaces to excite aircraft dynamic response in all six degrees of freedom simultaneously, while keeping the aircraft close to chosen reference flight conditions. Each maneuver was designed for a specific modeling task that cannot be adequately or efficiently accomplished using conventional flight test maneuvers. Each maneuver is described and explained, then demonstrated on a subscale jet transport aircraft in flight. Real-time and post-flight modeling results from equation-error parameter estimation in the frequency domain were used to show the effectiveness and efficiency of the maneuvers, as well as the quality of the aerodynamic models that can be identified from the resultant flight data.
\end{abstract}

\begin{tabular}{|c|c|}
\hline & Nomenclature \\
\hline AirSTAR & $=$ Airborne Subscale Transport Aircraft Research \\
\hline$b$ & $=$ wing span, $\mathrm{ft}$ \\
\hline $\bar{c}$ & $=$ wing mean aerodynamic chord, $\mathrm{ft}$ \\
\hline$C_{X}, C_{Y}, C_{Z}$ & $=$ body-axis nondimensional aerodynamic force coefficients \\
\hline$C_{l}, C_{m}, C_{n}$ & $=$ body-axis nondimensional aerodynamic moment coefficients \\
\hline GPS & $=$ global positioning system \\
\hline$I_{x}, I_{y}, I_{z}, I_{x z}$ & $=$ mass moments of inertia, slug- $\mathrm{ft}^{2}$ \\
\hline$m$ & $=$ aircraft mass, slug \\
\hline$p, q, r$ & $=$ body-axis roll, pitch, and yaw rates, $\mathrm{rad} / \mathrm{sec}$ or $\mathrm{deg} / \mathrm{sec}$ \\
\hline$S$ & $=$ wing reference area, $\mathrm{ft}^{2}$ \\
\hline$T$ & $=$ maneuver length, sec \\
\hline$V$ & $=$ airspeed, $\mathrm{ft} / \mathrm{sec}$ \\
\hline$\alpha$ & $=$ angle of attack, rad or deg \\
\hline$\beta$ & $=$ sideslip angle, rad or deg \\
\hline$\delta_{e}, \delta_{a}, \delta_{r}$ & $=$ elevator, aileron, and rudder deflections, rad or deg \\
\hline$\delta_{a_{l}}, \delta_{a_{r}}$ & $=$ left and right aileron deflections, rad or deg \\
\hline$\delta_{f}, \delta_{s}$ & $=$ trailing-edge flap and spoiler deflections, rad or deg \\
\hline$\varphi, \theta, \psi$ & $=$ Euler roll, pitch, and yaw angles, rad or deg \\
\hline$\omega$ & $=$ frequency, $\mathrm{rad} / \mathrm{sec}$ \\
\hline \multicolumn{2}{|l|}{ subscripts } \\
\hline$c g$ & $=$ center of gravity \\
\hline$l$ & $=$ lower \\
\hline$l i$ & $=$ left inboard \\
\hline lo & $=$ left outboard \\
\hline$o$ & $=$ reference value or base term \\
\hline$r i$ & $=$ right inboard \\
\hline ro & $=$ right outboard \\
\hline$u$ & $=$ upper \\
\hline
\end{tabular}

\footnotetext{
${ }^{1}$ Research Engineer, Dynamic Systems and Control Branch, MS 308, Associate Fellow

1 


\section{Introduction}

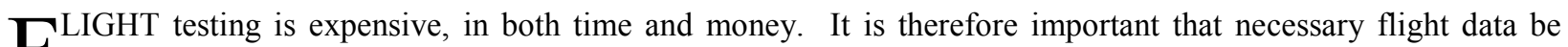
Collected in the most efficient manner possible. Inputs traditionally used for flight test maneuvers to collect aircraft stability and control data include doublets, impulses (stick raps), multi-steps, and frequency sweeps ${ }^{1}$. All of these input types are designed for single-axis response, although often the inputs are applied sequentially to different controls to collect multi-axis data.

Recently, an input design technique has been developed that combines the time efficiency of multi-axis excitation with optimized (minimum) input amplitudes, wide-band frequency content, and multiple-input orthogonality in both the time domain and the frequency domain ${ }^{1-6}$. This development in flight test input design has enabled new approaches to efficient stability and control flight testing that were previously not possible. Some of the problems that can be addressed are: multi-axis stability and control flight testing in off-nominal conditions such as high sideslip angles, near stall and departure, in post-stall flight, and during recovery from stalls and departures; multi-axis aerodynamic modeling over large portions of the flight envelope with a single maneuver; real-time monitoring of aircraft stability and control; efficient flight envelope expansion; and simultaneously estimating control effectiveness for a large number of individual control surfaces. The input design technique has been applied successfully to hypersonic stability and control flight testing ${ }^{4,5}$, real-time dynamic modeling for experimental fighter aircraft and subscale aircraft ${ }^{2,6}$, and as part of an envelope protection system for a commuter aircraft in icing conditions $^{7-9}$.

In this paper, novel maneuvers for efficient stability and control flight testing are described and demonstrated. All of the maneuvers are based on using the orthogonal optimized multi-sine input design technique described in detail in Refs. [1]-[6]. This basic input design technique is applied to collect data for a variety of aircraft dynamic modeling applications. The next section explains the basic input design technique. Following that is a brief description of the test aircraft and flight data. Then individual sections describe applications of the input design technique in novel ways for a variety of experimental investigations that are beyond usual flight testing situations.

All of the experiment design, data analysis, and modeling tasks included in the paper were done using system identification software written in MATLAB ${ }^{\circledR}$, called System IDentification Programs for AirCraft, or SIDPAC ${ }^{1}$. SIDPAC is bundled with Ref. [1], and is therefore publicly available. The SIDPAC software toolbox was developed at NASA Langley, and is continually expanded and improved. SIDPAC has been applied successfully to a wide variety of flight and wind tunnel experiments at NASA Langley ${ }^{10}$, and is used at more than 80 institutions worldwide ${ }^{11}$.

All modeling results shown in this work were generated using an equation-error formulation in the frequency domain $^{1,12}$. Ref. [1] provides a thorough description of this method (among others), along with information on how the method can be applied in practice and the MATLAB ${ }^{\circledR}$ software that implements it. Using equation-error in the frequency domain has been shown ${ }^{12}$ to produce modeling results comparable in accuracy to using iterative nonlinear optimization in a conventional output-error approach in the time domain, but requires much less computation and therefore is faster and easier to use. In fact, many of the modeling results presented here were computed in real time during the flight tests, using a real-time formulation of equation-error parameter estimation in the frequency domain ${ }^{1,6,13}$. The parameter estimates and error bounds shown are the real time estimates at the end of the maneuver, when data information content from the entire maneuver had been incorporated to produce the results. Because the flight testing used multi-axis excitations and a very fast real-time parameter estimation algorithm, it was possible to estimate stability and control derivatives for all 6 degrees of freedom in real time during all of the maneuvers described here.

Each experimental investigation is demonstrated using flight data from a subscale jet transport aircraft. Modeling results are used to show the effectiveness and efficiency of the flight test maneuvers in producing flight data that can be used to generate high-fidelity aerodynamic modeling results.

\section{Orthogonal Optimized Multi-Sine Input Design}

This section describes how orthogonal optimized multi-sine inputs are designed and why this particular input form is efficient for estimating stability and control parameters in dynamic models. More details on the input design method and flight applications can be found in Refs. [1]-[6].

The general idea is to excite the aircraft using perturbation inputs with wideband frequency content over a range of frequencies that encompasses the expected modal frequencies of the aircraft dynamic response. The excitations are implemented as perturbations to the control surface deflections by summing designed perturbation inputs with 
the actuator commands from the pilot and feedback control system, just before the actuator limiting on command rate and position.

Each designed perturbation input is a sum of sinusoids with unique frequencies, optimized phase shifts, and specified power distribution. Component frequencies are selected to cover a frequency band of interest, similar to frequency sweeps. The wide-band frequency content of the inputs is important because there is naturally some uncertainty as to what the modal frequencies are for the aircraft in flight. Wide-band inputs provide robustness to this uncertainty. Phase shifts for the sinusoidal components of each input are optimized to achieve low peak-to-peak amplitude and high input energy content for the sum of sinusoids. Amplitudes of the individual sinusoidal components can be chosen to achieve a specific power distribution.

Multiple inputs are designed to be mutually orthogonal in both the time domain and the frequency domain, and are optimized for maximum data information content in multiple axes over a short time period, while minimizing excursions from the nominal flight condition. The mutual orthogonality of the inputs allows simultaneous application of multiple inputs, which helps to minimize excitation time, but more importantly for this work, provides continuous multi-axis excitation as the aircraft

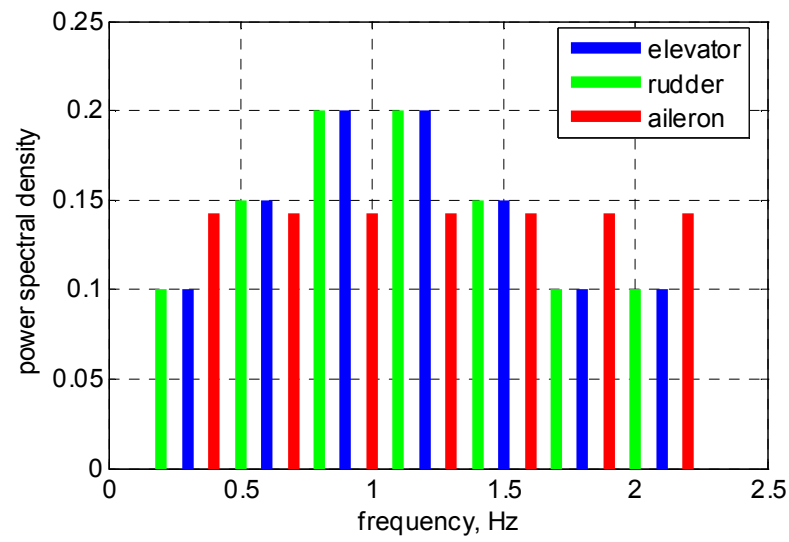

Figure 1. Multiple orthogonal phase-optimized multi-sine input spectra flies through time-varying or precarious flight conditions.

Each perturbation input $\boldsymbol{u}_{j}$, which is to be applied to the $j^{\text {th }}$ individual control surface, is comprised of a set of summed harmonic sinusoids with individual phase shifts $\phi_{k}$,

$$
\boldsymbol{u}_{j}=\sum_{k \in\{1,2, \ldots, M\}} A_{k} \sin \left(\frac{2 \pi k \boldsymbol{t}}{T}+\phi_{k}\right) \quad j=1,2, \ldots, m
$$

where $M$ is the total number of available harmonically-related frequencies, $T$ is the time length of the excitation, and $A_{k}$ is the amplitude for the $k^{\text {th }}$ sinusoidal component. The variable $t$ represents a vector of $N$ discrete time points, $\boldsymbol{t}=\left[\begin{array}{lllll}t(0) & t(1) & \ldots & t(N-1)\end{array}\right]^{T}$, and $\boldsymbol{u}_{j}$ represents the vector of corresponding amplitudes for the $j^{\text {th }}$ input, $\boldsymbol{u}_{j}=\left[\begin{array}{llll}u_{j}(0) & u_{j}(1) & \ldots & u_{j}(N-1)\end{array}\right]^{T}$. Each of the $m$ inputs is comprised of selected components from the pool of $M$ harmonic sinusoids with frequencies $\omega_{k}=2 \pi k / T, k=1,2, \ldots, M$, where $\omega_{M}=2 \pi M / T$ represents the upper limit of the frequency band for the excitation inputs. The interval $\left[\omega_{1}, \omega_{M}\right] \mathrm{rad} / \mathrm{s}$ specifies the range of frequencies where the aircraft dynamics are expected to lie.

If the phase angles $\phi_{k}$ in Eq. (1) were chosen at random on the interval $(-\pi, \pi] \mathrm{rad}$, then in general, the various harmonic components would add together at some points to produce an input $\boldsymbol{u}_{j}$ with relatively large amplitude excursions. This is undesirable, because it can result in the dynamic system being moved too far from the reference condition selected for the experiment. To prevent this, the phase angles $\phi_{k}$ for each of the selected harmonic components are chosen to minimize relative peak factor $R P F^{1}$, defined by

$$
R P F\left(\boldsymbol{u}_{j}\right)=\frac{\left[\max \left(\boldsymbol{u}_{j}\right)-\min \left(\boldsymbol{u}_{j}\right)\right] / 2}{\sqrt{2\left(\boldsymbol{u}_{j}^{T} \boldsymbol{u}_{j}\right) / N}}=\frac{\left[\max \left(\boldsymbol{u}_{j}\right)-\min \left(\boldsymbol{u}_{j}\right)\right]}{2 \sqrt{2} \operatorname{rms}\left(\boldsymbol{u}_{j}\right)}
$$

Relative peak factor is a measure of the efficiency of an input for dynamic modeling purposes, in terms of the amplitude range of the input divided by a measure of the input energy. The relative peak factor is scaled so that any 
individual sinusoidal component (such as any one of the summands in Eq. (1)) has $R P F=1$. Low relative peak factors are desirable and efficient for estimating dynamic model parameters, because the objective is to excite the dynamic system with good input energy over a variety of frequencies while minimizing the input amplitudes in the time domain, to avoid driving the dynamic system too far away from the reference condition.

For a composite signal with more than one sinusoidal component, as in Eq. (1), the goal of designing an input with minimum $R P F$ is achieved by adjusting the phase parameters $\phi_{k}$ for the sinusoidal components of the input. The resulting optimization problem is non-convex; however, a simplex algorithm ${ }^{1,14}$ can be applied to find a solution.

The integers $k$ specifying the frequencies for the $j^{\text {th }}$ input $\boldsymbol{u}_{j}$ are selected to be unique to that input, but are not necessarily consecutive. A good approach for multiple inputs is to assign integers $k$ to each input alternately. This is illustrated in Figure 1 for a flight test maneuver design on the T-2 aircraft. In that case, there were 3 inputs: elevator, rudder, and aileron, and a total of 21 frequencies $(M=21)$. The frequencies were interleaved among the three inputs to achieve wide-band frequency content for each input. This provided robustness to uncertainty in how each control excites the dynamic modes of the aircraft. Because each input has wide-band frequency content, the same input design can be applied at various flight conditions, which simplifies the flight test and reduces flight computer memory requirements. It is even possible to use the same input design for different aircraft, because of the wide-band frequency content of the excitation inputs. Table 1 contains the information needed to assemble the input design shown in Figure 1, using Eq. (1).

To achieve a uniform power distribution, the $A_{k}$ are selected as

$$
A_{k}=\frac{A}{\sqrt{n}} \quad \forall k
$$

where $n$ is the number of sinusoidal components included in the summation of Eq. (1), and $A$ is the amplitude of the composite input $\boldsymbol{u}_{j}$. Therefore, with uniform power distribution, selection of the $A_{k}$ reduces to selecting a single value for the input amplitude $A$. Each input $\boldsymbol{u}_{j}$ can of course have arbitrary amplitude $A$, subject to practical flight testing and modeling constraints.

In Figure 1, the power spectra for elevator and rudder were modified so that more excitation power was applied at middle frequencies where the natural frequencies of the dynamic modes excited by these controls were believed to be. For each input, the power spectrum can be tailored by selecting the $A_{k}$ in Eq. (1) to distribute power over the spectral components. The power spectra shown in Figure 1 are normalized, so the effects of individual control surface amplitudes are excluded. This means that for each input, the sum of all the spectral line ordinates (sum of the heights of the bars for each input) is 1.

When the frequency indices $k$ selected for each input $\boldsymbol{u}_{j}$ in Eq. (1) are distinct from those chosen for the other inputs, then the frequency content of each $\boldsymbol{u}_{j}$ consists of distinct spectral lines in the frequency domain, as can be seen in Figure 1. Therefore, the vectors of Fourier transforms for the inputs as a function of frequency have inner products equal to zero. In this sense, the inputs are mutually orthogonal in the frequency domain, because each input contains frequencies that no other input has.

In the time domain, a sum of sinusoids is orthogonal to any other sum of sinusoids with harmonically-related frequencies, regardless of the constant phase shift of each sinusoidal component ${ }^{1,5}$. This means the inputs are also mutually orthogonal in the time domain.

An objective for the experiment design is to excite the aircraft dynamics in all axes over a short time period by moving multiple control surfaces simultaneously. This is particularly important in situations where the reference flight condition cannot be maintained for very long, or is time-varying. 

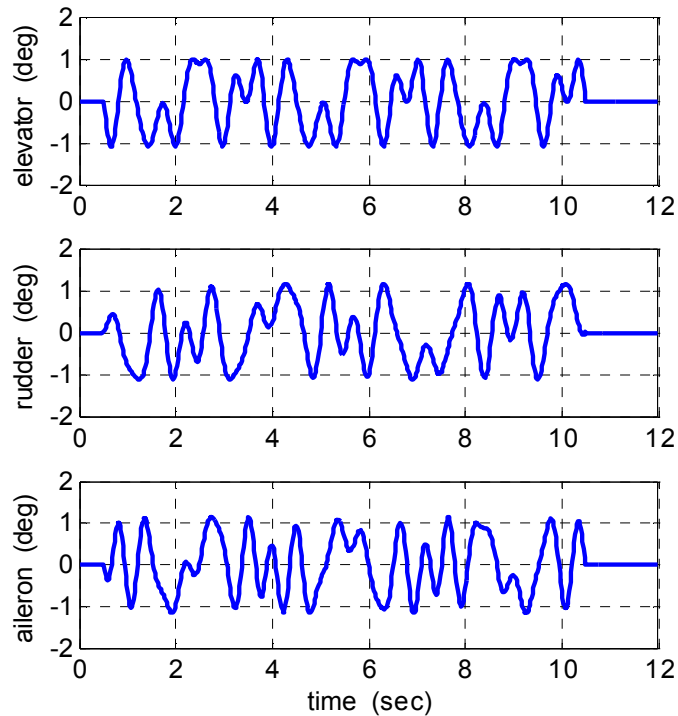

Figure 2. Multiple orthogonal phase-optimized multi-sine inputs

Since more than one surface is being moved, it is advantageous for modeling purposes if the $\boldsymbol{u}_{j}$ applied to the control surfaces are mutually orthogonal. This helps the dynamic modeling by completely de-correlating the inputs, which improves the accuracy of control effectiveness estimates. Using the input design method described here, it is possible to make all of the $\boldsymbol{u}_{j}$ mutually orthogonal in both the time and frequency domains, while also minimizing relative peak factor for each $\boldsymbol{u}_{j}$, which keeps the aircraft from departing significantly from the reference flight condition. This gives the analyst the flexibility to use either time domain or frequency domain parameter estimation methods while retaining the desirable feature of mutually orthogonal inputs.

Figure 2 shows the perturbation input time series for the maneuver design specified in Figure 1 and Table 1. These inputs are mutually orthogonal in both the time and frequency domains. The inputs were computed from Eq. (1) and Table 1, with phase angles $\phi_{k}$ optimized for minimum relative peak factor. Because of the various frequencies and phase angles, and the small amplitudes of the perturbation inputs, applying these inputs simultaneously to the aircraft produces a dynamic response similar to what might be seen in flight through light to moderate turbulence. Consequently, the aircraft stays near the reference condition, but jiggles around that. In practice, pilot inputs and feedback control can act to ruin the input orthogonality; however, good modeling results require only low correlations, not zero correlations, so that slightly imperfect inputs still work quite well.

\section{III.T-2 Subscale Jet Transport Aircraft}

\section{Airframe}

The T-2 aircraft is a 5.5 percent dynamically-scaled model of a generic commercial twin-engine jet transport aircraft. Figure 3 shows a photograph of the aircraft in flight. The aircraft has twin jet engines mounted under the wings and retractable tricycle landing gear. Aircraft geometry and nominal mass properties are given in Table 2. Further information on the T-2 subscale jet aircraft and associated flight test operations can be found in Refs. [15]-[18]. A similar airframe was tested extensively in the wind tunnel ${ }^{19}$, although the wind tunnel model differed in some geometric details from the aircraft used for the flight tests. The wind tunnel data provided a reference for comparison with the aerodynamic models identified directly from flight data.

\section{Control Surfaces}

Control surfaces on the T-2 aircraft are left and right ailerons, left and right inboard and outboard elevators, upper and lower rudders, left and right inboard and outboard trailing-edge flaps, and left and right inboard and outboard spoilers, for a total of 16 independent control surfaces. For most of the data analyzed in this work, only the elevators, ailerons, and rudders were deflected. The individual elevator surfaces were moved together as a single elevator surface, and similarly for the rudders. Left and right ailerons were deflected asymmetrically, in the conventional way. In some cases, the control surfaces were deflected individually and

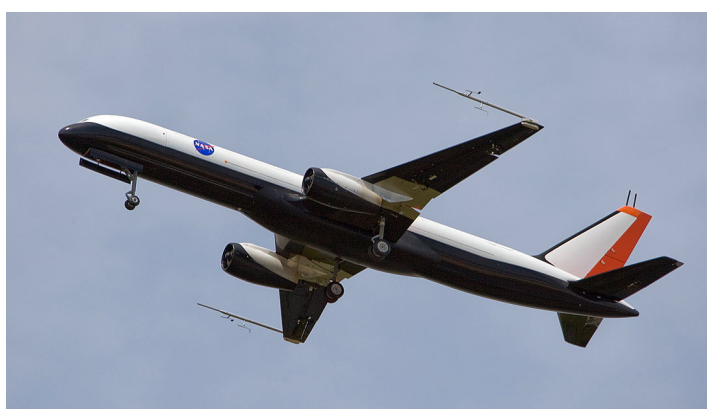

Figure 3. T-2 Subscale Jet Transport Aircraft Credit: NASA Langley Research Center independently. This will be pointed out specifically in context. Definitions of control surface deflections are given below. Trailing edge down is positive deflection for wing and elevator surfaces, and trailing edge left is positive for rudder surfaces. 


$$
\begin{gathered}
\delta_{e}=\frac{1}{4}\left(\delta_{e_{l o}}+\delta_{e_{l i}}+\delta_{e_{r i}}+\delta_{e_{r o}}\right) \\
\delta_{a}=\frac{1}{2}\left(\delta_{a_{r}}-\delta_{a_{l}}\right) \quad \delta_{r}=\frac{1}{2}\left(\delta_{r_{u}}+\delta_{r_{l}}\right)
\end{gathered}
$$

The aircraft can be flown by a safety pilot using direct visual contact and conventional radio control. A research pilot executed the flight test maneuvers from inside a mobile control room, using a synthetic vision display drawn from telemetry data and a local terrain database, along with video from a camera in the nose of the aircraft. Inputs from the research pilot and a ground-based flight control system were used to generate control surface commands which were transmitted by telemetry to the aircraft.

The flight control system has the capability to inject automated control surface perturbations to excite the aircraft dynamic response for modeling purposes. These control surface perturbations can have arbitrary waveforms, and can be applied to multiple control surfaces individually or simultaneously. The perturbations are summed with pilot and feedback control commands in the flight control system, just before the actuator command rate and position limiting. Typically, the research pilot flies the aircraft to the desired flight condition(s), then initiates the automated control surface perturbations with a trigger switch on the control stick.

Failure and damage conditions, such as a stuck control surface, reduced control effectiveness, reduced aircraft stability, or inoperative sensor(s), can be emulated in software by the flight control computer ${ }^{17}$.

\section{Instrumentation and Data Acquisition}

The T-2 aircraft was equipped with a micro-INS, which provided 3-axis translational accelerometer measurements, angular rate measurements, estimated attitude angles, and GPS velocity and position. Air data probes attached to booms mounted on each wingtip (visible in Figure 3) measured angle of attack, sideslip angle, static pressure, and dynamic pressure. Measurements from static pressure sensors and ambient temperature sensors were used to compute air density and altitude. Engine speeds in rpm were measured and used as inputs to an engine model to compute thrust. The engine model was identified from ground test data, with adjustments for ram drag identified from flight data. Potentiometers on the rotation axes of the control surfaces were used to measure control surface deflections. Mass properties were computed based on measured fuel flow, pre-flight weight and balance, and inertia measurements done on the ground for the aircraft without fuel. The pilot stick and rudder pedal commands and throttle position were also measured and recorded. Data from onboard sensors were telemetered to the ground in real time. Sampling rate for the flight data was $200 \mathrm{~Hz}$, decimated to $50 \mathrm{~Hz}$ for data analysis and modeling.

\section{Flight Test Maneuvers}

The flight test maneuvers to be discussed in the following subsections involved application of multiple orthogonal optimized multi-sine inputs in various situations for stability and control flight testing on the T-2 subscale aircraft.

\section{A. Aircraft Stability and Control at High Sideslip Angle}

Steady flight at a high nominal sideslip angle required the pilot to hold in rudder while applying aileron control to compensate for the aircraft tendency to roll because of the aircraft inherent static lateral stability $C_{l_{\beta}}$. In this flight condition, it would be extremely difficult for a pilot to execute a dynamic maneuver for collecting modeling data without departing from the desired flight condition. However, with automated control surface perturbations implementing multiple orthogonal optimized multi-sine inputs, collecting dynamic data in this flight condition was very efficient and straightforward. The pilot held controls for steady level flight at high nominal sideslip angle of approximately $8 \mathrm{deg}$, then initiated the automated orthogonal optimized multi-axis perturbation inputs with a trigger on the control stick. Figure 4 shows flight data from the maneuver on the T-2 aircraft. Good excitation was achieved in all axes simultaneously, with low pilot workload. 

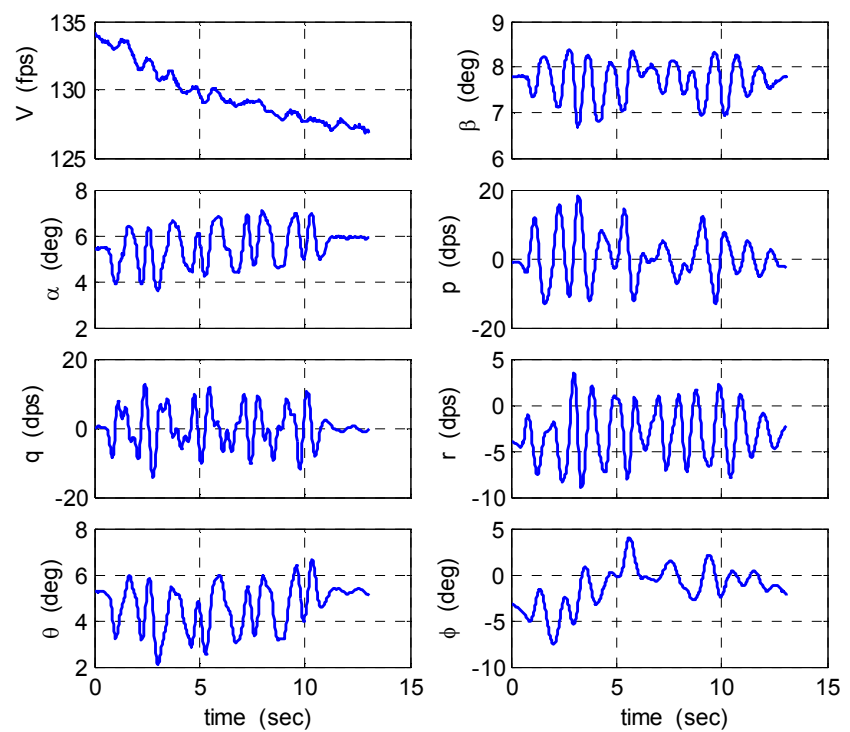

Figure 4. T-2 flight data for an orthogonal optimized multi-axis maneuver at high nominal sideslip angle

Figure 5 shows the coverage of angle of attack and sideslip angle during the maneuver, where the $\mathrm{x}$ symbols indicate individual data points. The maneuver generated data that covered the targeted range of angle of attack and sideslip angle very well while also providing a wide variety of combinations of high and low values for each of these important explanatory variables, and similarly for the other explanatory variables. Throughout the dynamic maneuver, the aircraft remained close to the nominal flight condition, allowing the use of a simple linear model structure for the aerodynamic modeling. Data from this maneuver produced highly accurate parameter estimates, as shown in Figure 6. The triangle markers in Figure 6 indicate the estimated parameter values, and the associated error bars represent the 95 percent confidence $( \pm 2$ standard errors) intervals. Note that only a subset of the estimated parameters are shown in Figure 6. Stability and control parameters for all 6 degrees of freedom were estimated simultaneously from this single maneuver. Other stability and control parameters were estimated with similar accuracy.

\section{B. Aircraft Stability and Control at Post-Stall Angle of Attack}

The T-2 aircraft can be flown to post-stall angles of attack using a timed combination of full aft pilot stick deflection and increased engine power level. However, this flight condition can be maintained for only a short time, because of test range limitations and rapid altitude loss. In this situation, both the short time available and the precarious nature of the flight condition were addressed by applying orthogonal optimized multi-sine perturbation inputs. Orthogonal multi-axis excitation provided the necessary time efficiency, and the optimized minimum amplitudes of the perturbations kept the aircraft near the reference condition throughout the maneuver.

Figure 7 shows flight data from this maneuver. The pilot put the aircraft into a steady flight condition at approximately 20 deg angle of attack (normal power-off stall angle of attack was $14 \mathrm{deg}$ ), then held controls steady while applying the automated orthogonal optimized multi-axis perturbation inputs with a trigger on the control stick. Note that significant high-frequency content in the lateral acceleration signal was associated with high angle of attack, and not with thrust level. Figure 8 shows the variation in angle of attack and sideslip angle during the maneuver, and Figure 9 shows selected parameter estimation results at the post-stall angle of attack flight condition.

Accurate estimates were achieved for model parameters in all axes simultaneously. These results would not be possible using conventional flight test methods. 

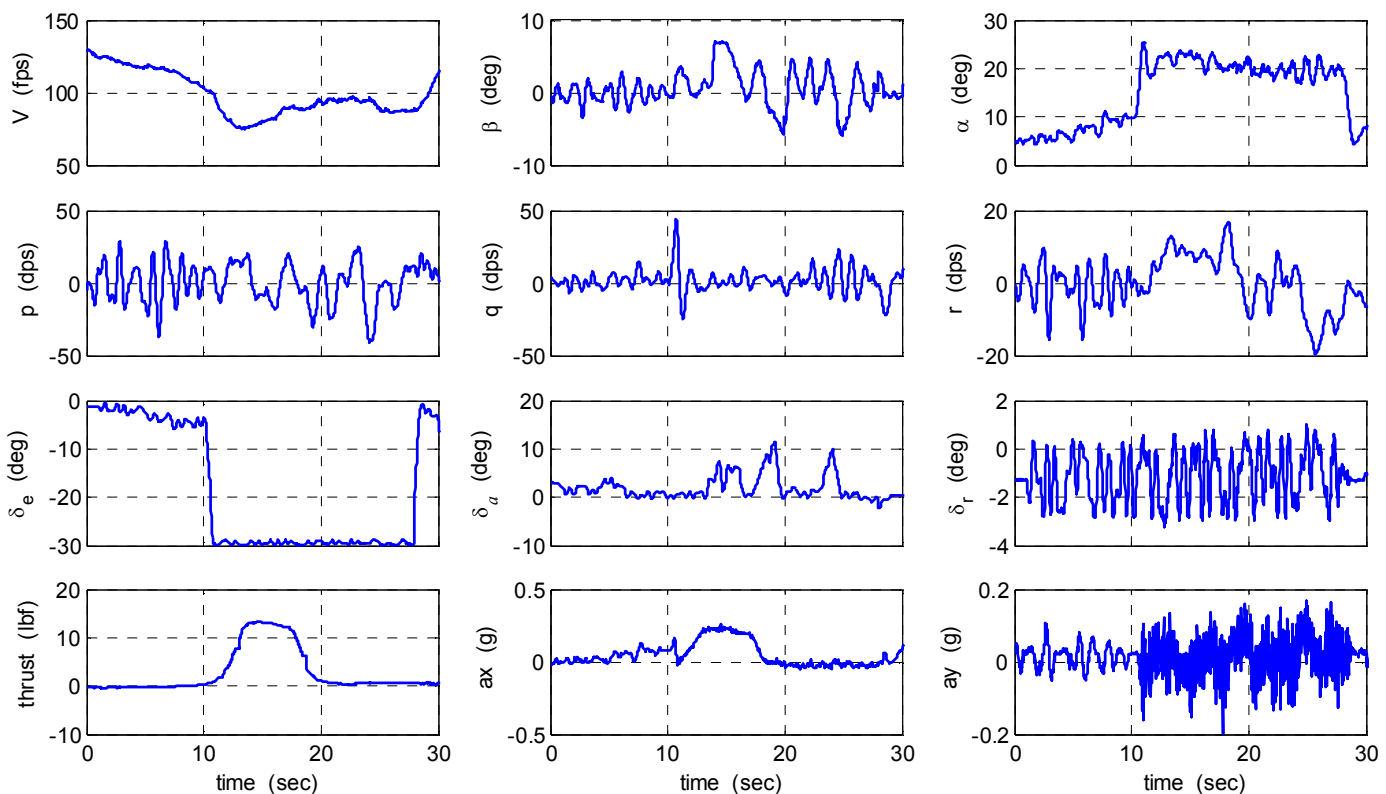

Figure 7. T-2 flight data for an orthogonal optimized multi-axis maneuver at post-stall angle of attack

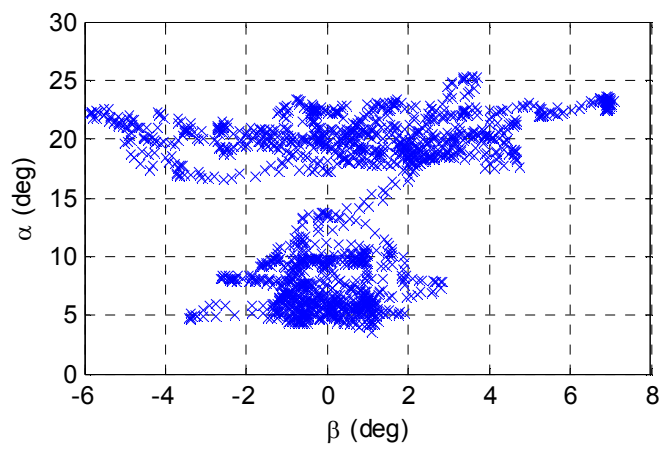

Figure 8. Explanatory variable coverage for T-2 maneuver at post-stall angle of attack
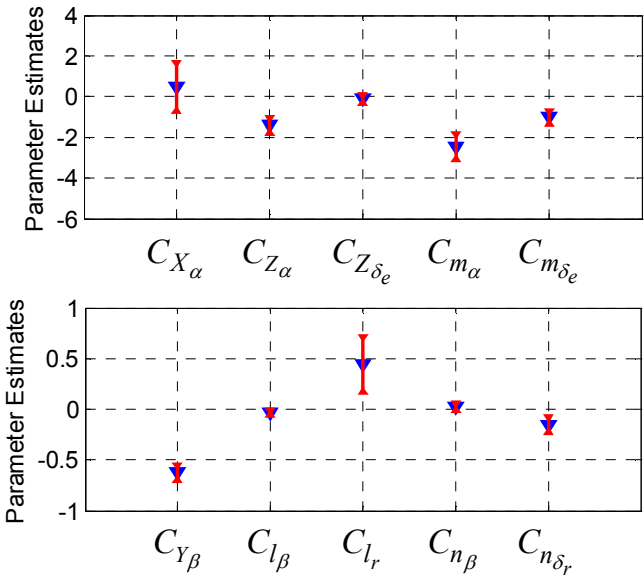

Figure 9. Parameter estimation results from T-2 maneuver at post-stall angle of attack

\section{Efficient Global Aerodynamic Modeling}

The previous two subsections described flight test experiments where optimized perturbation inputs such as those shown in Figure 2 were applied at a selected unusual and precarious flight condition. The pilot flew the aircraft to the reference flight condition, then held that flight condition while activating an automated excitation system that added inputs like those shown in Figure 2 to the control deflections coming from the pilot and the feedback control system. This can be seen clearly in the elevator deflection plot in Figure 7. The flight data generated was excellent for aerodynamic parameter estimation at the selected flight condition. This approach could be called local aerodynamic modeling in unusual flight conditions.

For global aerodynamic modeling, one conventional approach is to combine local aerodynamic modeling results obtained from local perturbation maneuvers to produce a global aerodynamic model. This requires numerous and accurate acquisitions of a particular flight condition, followed by the application of perturbation excitations. 
More efficient global aerodynamic modeling can be achieved by continuously applying multi-axis perturbation inputs while the aircraft flight condition is varied slowly. This approach is practical because the orthogonal optimized multi-axis perturbations excite the aircraft dynamics in a very time-efficient manner with high data
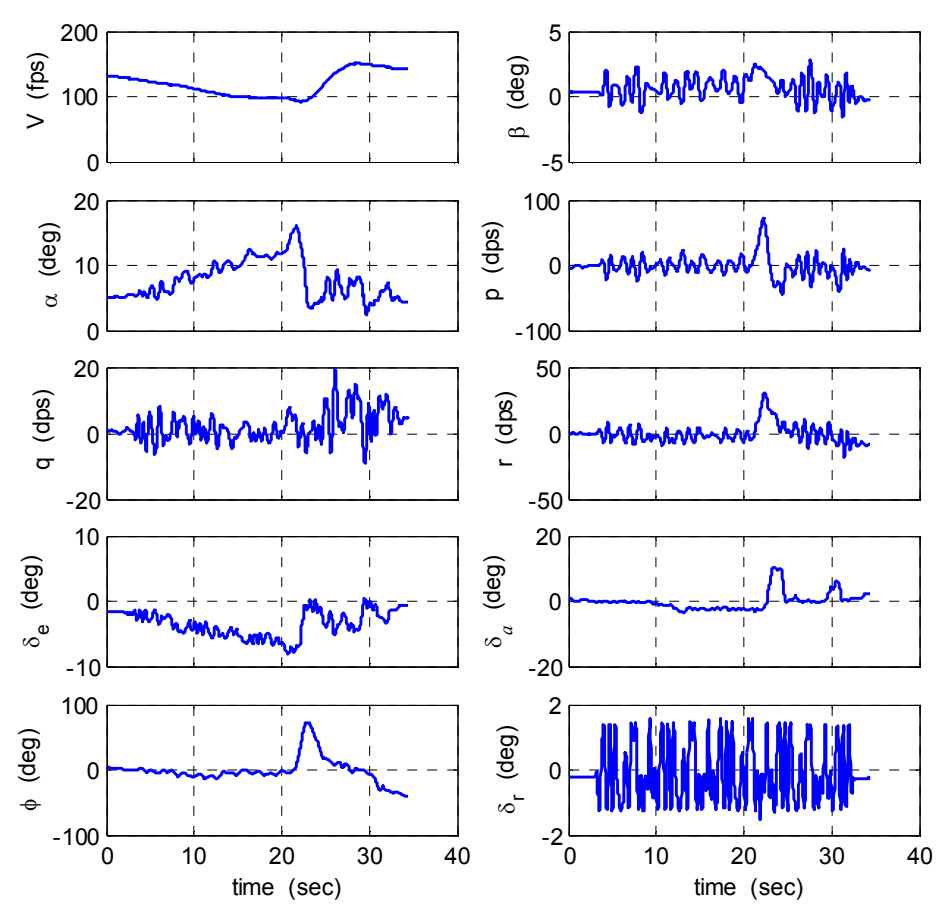

Figure 10. T-2 flight data for an orthogonal optimized multi-axis maneuver while slowly varying angle of attack information content, so that the aircraft dynamics can be sufficiently excited even when the flight condition is changing.

For this maneuver, the pilot began with a steady wings-level trim condition at low angle of attack, then initiated multiaxis excitation inputs like those shown in Figure 2. As the excitation inputs were being applied, the pilot pulled back slowly on the stick to induce a slow increase in the nominal angle of attack. Multi-axis excitation inputs were continuously applied additively to the control surface deflections commanded by the pilot and feedback control system. The pilot inputs in lateral stick and rudder pedal were essentially zero, but the elevator deflection commanded by the pilot changed slowly to implement the slow increase in nominal angle of attack. An example of the resulting flight data is shown in Figure 10. This maneuver produces very informative data over a wide range of nominal angle of attack. Only subsonic aerodynamics at relatively low altitude were being studied, so the effects of changing airspeed (or Mach number) were adequately modeled by conventional nondimensionalization using dynamic pressure. The multi-axis excitation was continued throughout the stall and recovery portions of the maneuver, providing stability and control information at these flight conditions as well. Since the nominal angle of attack changed slowly, this maneuver could be considered a combination of informative multi-axis excitation data for many different nominal angles of attack, executed in a single efficient combined maneuver.
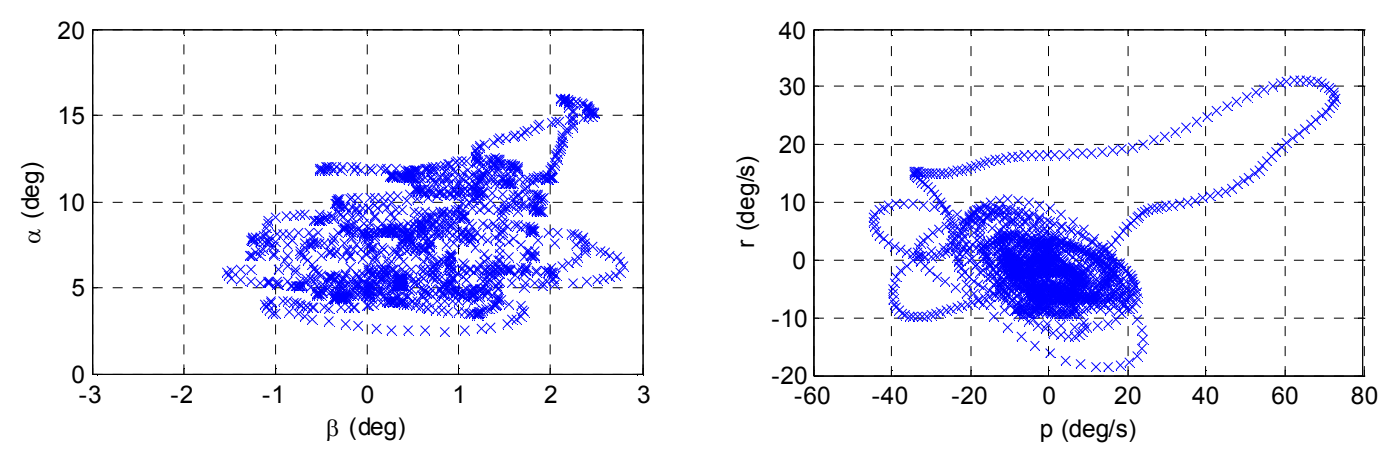

Figure 11. Explanatory variable coverage for T-2 maneuver while slowly increasing angle of attack through stall and recovery

Figure 11 shows cross-plots of aircraft states and controls using data from the maneuver shown in Figure 10. These plots demonstrate that a wide range of the explanatory variables generally used for aerodynamic modeling was swept through during this single maneuver. Note also that the cross-plots generally do not show anything close to diagonal lines or ellipses, which means that the explanatory variable data from this maneuver had very low pair- 
wise correlations. Low pair-wise correlations mean that the aerodynamic dependencies on the explanatory variables can be identified accurately and without ambiguity.

Several of these maneuvers were flown on the T-2 aircraft. Each maneuver had a constant power setting and aircraft configuration, but all used multi-axis perturbation time series, similar to what is shown in Figure 2. For each flight test deployment, the same perturbation inputs could be used for the maneuvers because the wide-band frequency content of the excitation inputs made them effective without modification for different power settings and aircraft configurations. The versatility of the excitation inputs was also helpful in practical implementation, because only one set of multi-axis perturbation time series had to be programmed and stored in the flight control computer. Table 3 lists the aircraft configuration and power setting for these maneuvers. For each flight test deployment, some variation in the excitation input design was introduced to study input frequency content, time length, and amplitude effects.

This novel maneuver exhibits a combination of low pair-wise correlations, multi-axis excitation, and slowlyvarying flight conditions that cover a large portion of the explanatory variable subspace for aerodynamic modeling. These characteristics make the maneuver very effective and efficient for global aerodynamic modeling. It is possible to use this data all at once to identify a single global aerodynamic model for each force or moment coefficient, or to partition the data according to angle of attack, and identify a series of local aerodynamic models, or to identify a series of local aerodynamic models based on a sliding time window of data. All of these modeling approaches are being pursued.

\section{Flap Effects in Landing Configuration}

A typical flight testing approach for collecting data to determine the effects of flap deflection on aircraft stability and control is to execute dynamic flight test maneuvers at various fixed flap deflections. Flight data for estimating flap effects can be obtained much more efficiently and comprehensively by varying flap deflection linearly in time while simultaneously exciting the aircraft dynamics using multiple orthogonal optimized inputs. This approach is similar to the global modeling maneuver, in that a particular explanatory variable, in this case flap deflection, is varied in a relatively simple and slowly-varying way, while automated optimized multi-axis excitations are applied continuously to the primary control surfaces to collect data for dynamic modeling.

Figure 12 shows aircraft control surface deflections and responses during the maneuver for estimating flap effects. The maneuver started with the aircraft in landing configuration with full flap deflection, then the flaps were retracted slowly while orthogonal optimized multi-sine excitation inputs were applied. The landing gear were deployed throughout the maneuver, so that the modeling results characterized flap effects in landing configuration. Figure 13 shows modeling results for the longitudinal aerodynamics. Accurate estimates were achieved for model parameters in all axes simultaneously, with model parameter estimate accuracies below 10 percent, except for the weak control derivatives $C_{X_{\delta_{e}}}, C_{Z_{\delta_{e}}}$, and $C_{m_{\delta_{f}}}$. The flap effectiveness was accurately identifiable because the ramp input on the flaps had very low correlation with the multi-axis excitation applied to the elevator, aileron, and rudder control surfaces and the resulting aircraft responses.

Determining flap effects in landing configuration was important operationally because there was high interest in the stability and control of the aircraft in landing configuration, to ensure safe approach and landing, to evaluate test range safety in case the aircraft switched into a fail-safe condition with flaps down, and also because of the desire to investigate the use of flaps for adaptive control in failure conditions. This maneuver determined the effects of all four flap control surfaces (left and right inboard and outboard) moved symmetrically at the same time. 

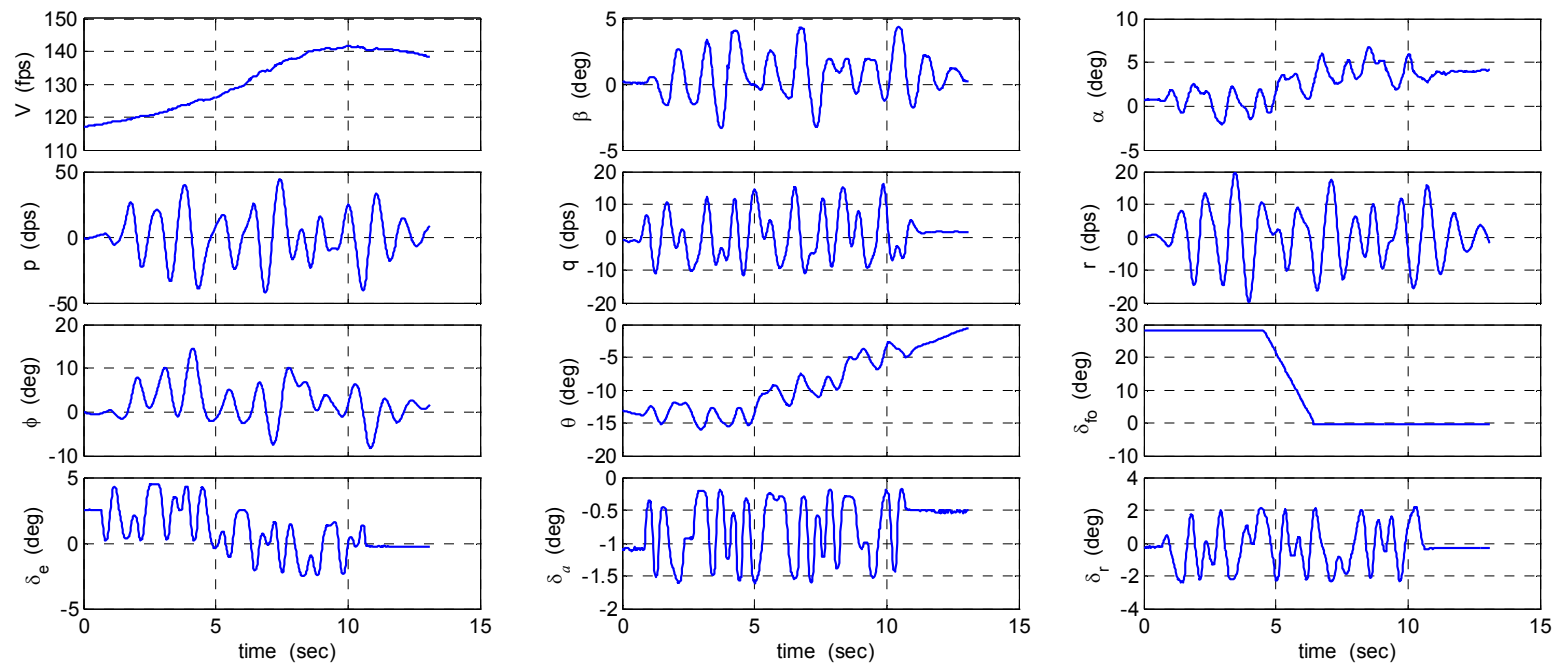

Figure 12. T-2 flight data for an orthogonal optimized multi-axis maneuver while slowly varying symmetric flaps in landing configuration
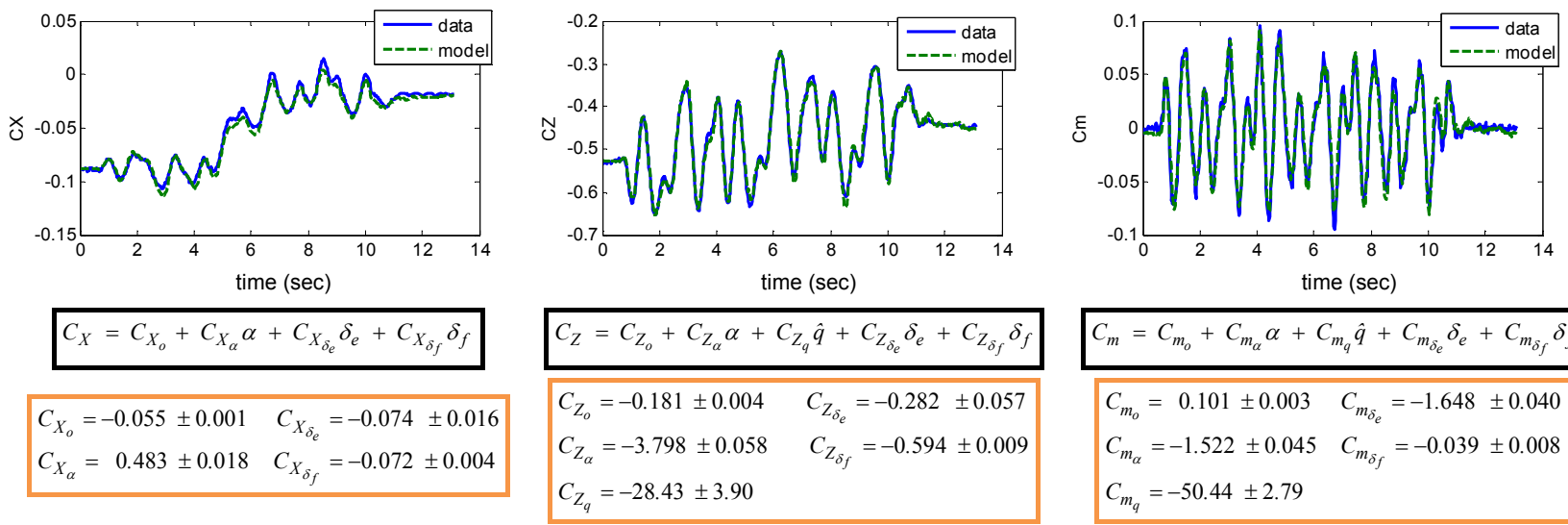

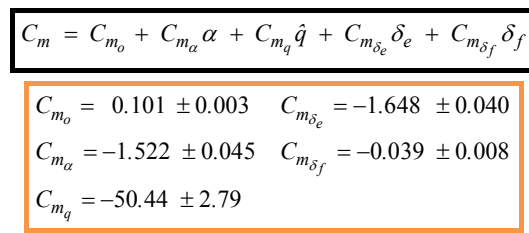

Figure 13. T-2 longitudinal modeling results for an orthogonal optimized multi-axis maneuver while slowly varying symmetric flaps in landing configuration

\section{E. Efficient Comparison of Flight Results to a Simulation Database}

Real-time comparisons of aerodynamic parameter estimates from flight data with analogous parameters extracted from a simulation database are useful for efficient flight envelope expansion and for evaluating the accuracy of the simulation database for predicting flight responses. To achieve this efficiently, orthogonal optimized multi-sine inputs can be applied at various flight conditions to excite the aircraft dynamics, and real-time parameter estimates ${ }^{1,13}$ can be compared with simulation values extracted in real time.

Figure 14 shows flight data for a maneuver using orthogonal optimized multi-sines at approximately 4.5 deg nominal angle of attack on the T-2 aircraft. Real-time parameter estimation in the frequency domain was applied to compute flight estimates of the aircraft stability and control derivatives. The real-time parameter estimation routine had no prior information on the values of the unknown parameters. At the end of the maneuver, the ranges of aircraft states and controls traversed during the maneuver were used to define finite difference perturbation sizes for computing aircraft stability and control derivatives from the simulation database. This provided a good comparison between the flight results and the simulation database. However, the values extracted from the simulation using finite differences were necessarily done by perturbing one state or control at a time, whereas the flight estimates 
were obtained from flight maneuver data where all states and controls moved simultaneously, but in different ways. This caused a slight mismatch in the actual quantities being compared, but this effect was considered small enough to be ignored for real-time comparisons.
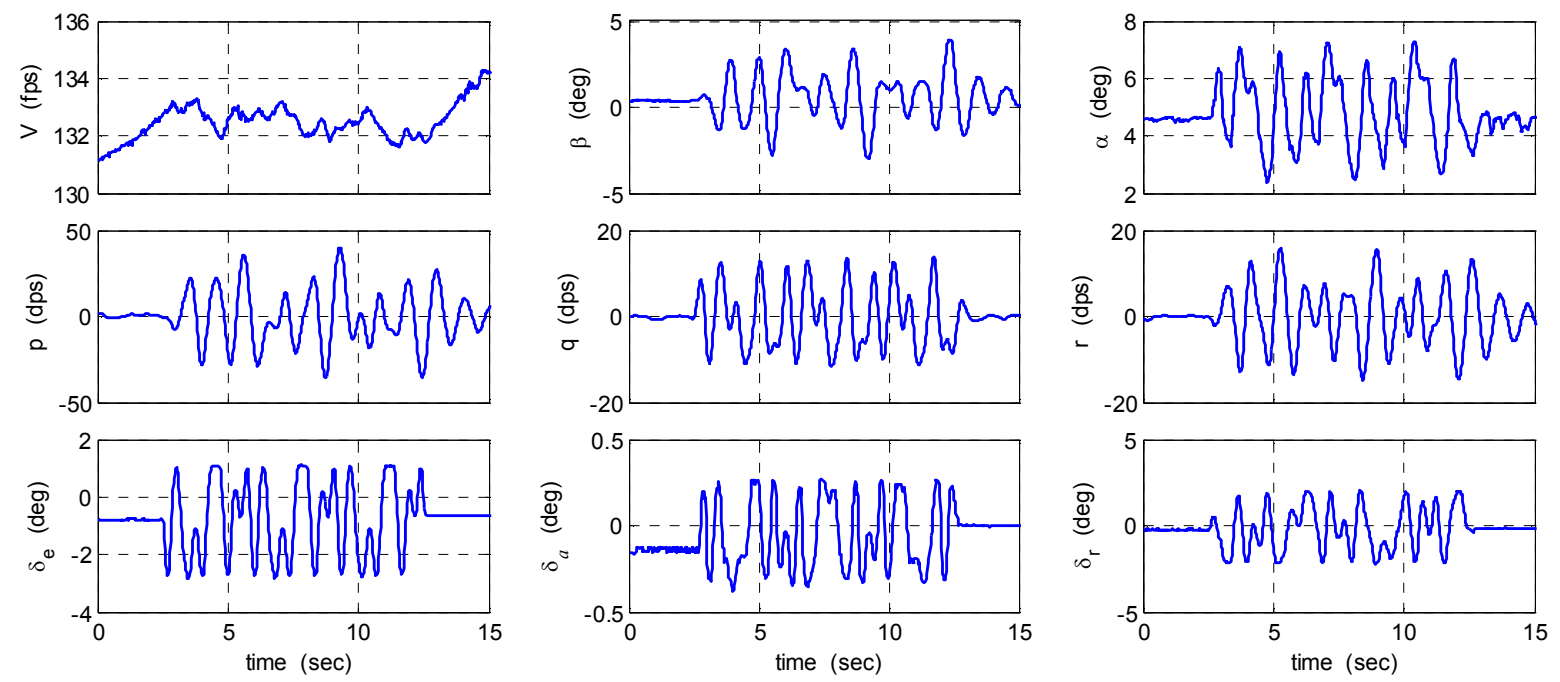

Figure 14. T-2 flight data for an orthogonal optimized multi-axis maneuver used for real-time flight-to-simulation comparisons

Figure 15 shows real-time comparisons of flight estimates for some selected nondimensional stability and control derivatives with corresponding finite difference values extracted from the simulation database, which was based on wind tunnel data ${ }^{19}$. The dashed lines indicate the simulation values, and the triangle markers indicate the real-time parameter estimates, with associated error bars representing the 95 percent confidence ( \pm 2 standard errors) intervals. In general, on-axis moment control derivatives such as $C_{m_{\delta_{e}}}$ and $C_{n_{\delta_{r}}}$ matched well, whereas the static and dynamic stability derivatives and weak control derivatives such as $C_{Z_{\delta_{e}}}$ exhibited a slightly degraded match. This general trend was consistent for the other stability and control derivative comparisons not shown. Using flight test maneuvers implemented by multi-axis orthogonal optimized multi-sine excitations, along with real-time interrogation of the simulation database and real-time parameter estimation from flight data, it was possible to achieve real-time comparisons of all stability and control derivatives. This demonstrated a very efficient flight test approach for simulator validation and flight envelope expansion. The practical challenge has now become having enough flight test personnel to watch and evaluate all of the many real-time stability and control parameter comparisons, or to develop an automated capability for evaluating the quality of the real-time comparisons.

\section{F. Multiple Control Surface Effectiveness}

Accurate flight estimates of individual control surface effectiveness are useful for many purposes, such as control system design, aerodynamic studies, comparisons with wind tunnel data, and developing in-flight simulation capabilities. Furthermore, modern aircraft are evolving toward using an increasing number of control surfaces and other aerodynamics-based effectors. This drives the need for efficiency in collecting modeling data for aerodynamic control effectors. Theoretically, the multi-input design method described earlier could be used to efficiently collect modeling data for any number of individual control effectors, all at the same time with a single maneuver. However, there are some practical limitations, which can be seen by studying the T-2 aircraft example presented here. 

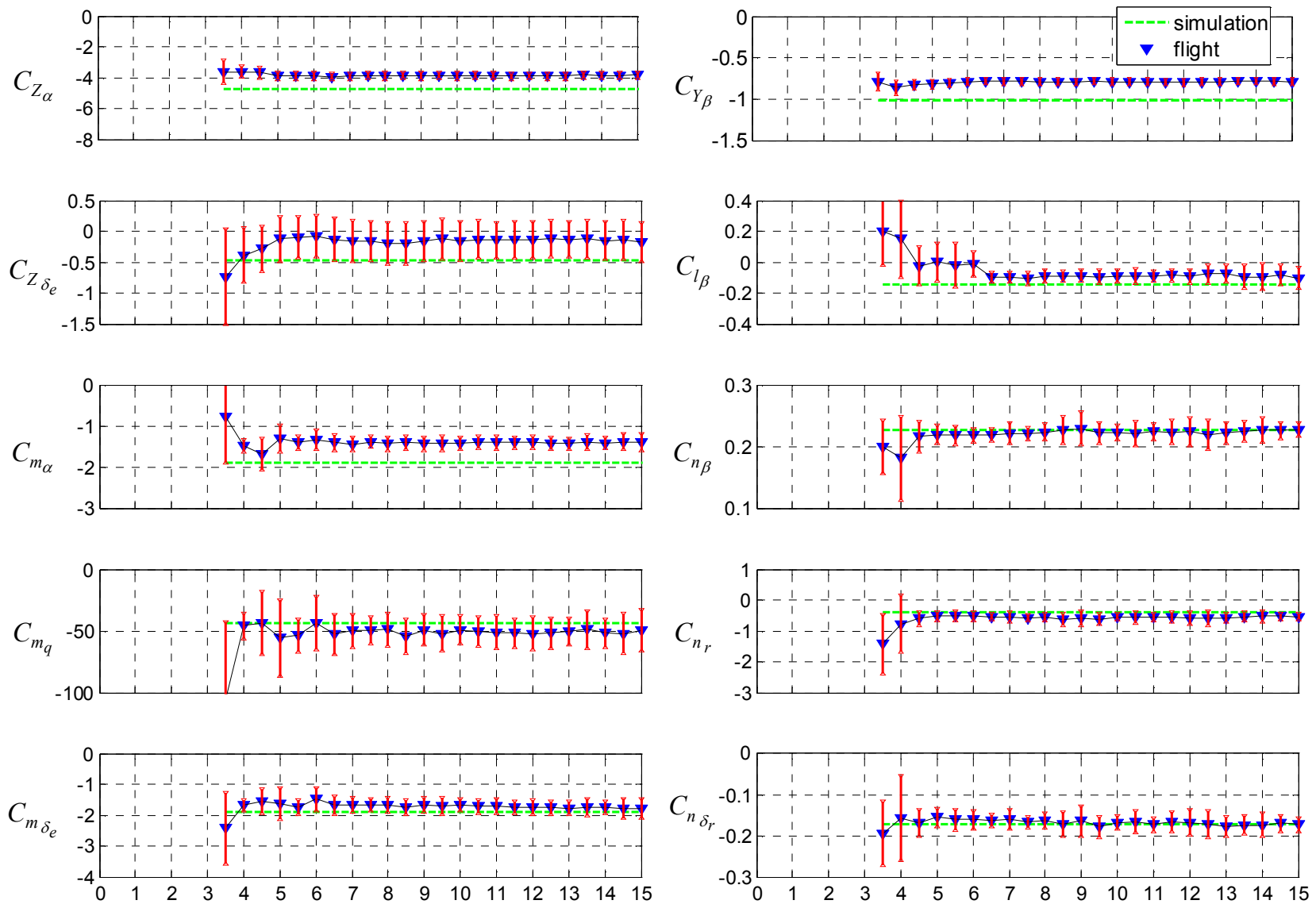

Figure 15. T-2 real-time flight test modeling comparisons with simulation wind-tunnel database

The T-2 aircraft has 16 individual control surfaces - two outboard elevators, two inboard elevators, two ailerons, left and right inboard and outboard flaps and spoilers, and upper and lower rudder. An input design diagram similar to Figure 1 could be drawn for a maneuver design with 16 individual control surfaces. Time length for the flight test maneuver was chosen as $40 \mathrm{sec}$, making the frequency resolution for harmonic sinusoids equal to $1 / 40 \mathrm{sec}$ or $0.025 \mathrm{~Hz}$. That was the minimum spacing between the individual frequency lines that could be assigned to the 16 individual control surfaces. In order to apply a wide range of frequencies to each individual control surface, the available frequencies were assigned to each of the 16 individual control surfaces in turn, similar to what is shown in Figure 1. For the T-2 aircraft, the design covered the frequency band $[0.1,1.675] \mathrm{Hz}$ with frequencies spaced at $0.025 \mathrm{~Hz}$. This made 64 frequencies available, or 4 frequencies for each of the 16 control surfaces. A longer maneuver time would give more closely spaced available frequencies, and therefore more frequencies available for each input over the same frequency range, or provide the ability to excite more control surfaces.

The flaps and spoilers differed from the other control surfaces in that the flaps and spoilers moved in one direction only, namely outward from flush with the aircraft wing. Because the perturbation inputs for these control surfaces would have only one sign, they could not be made orthogonal to the others, although it is still possible to design inputs with relatively low pair-wise correlations in that case. However, to retain the mutual orthogonality for all 16 individual control surfaces, the flaps and spoilers were initially deflected away from their flush position by $10 \mathrm{deg}$ as part of the process of flying the aircraft to the desired reference condition. This allowed two-sided perturbation inputs to be used for all 16 controls and retained the designed mutually orthogonality of all 16 individual control surfaces, for minimum (zero) pair-wise correlations and consequent high accuracy for the control surface effectiveness parameter estimates. 

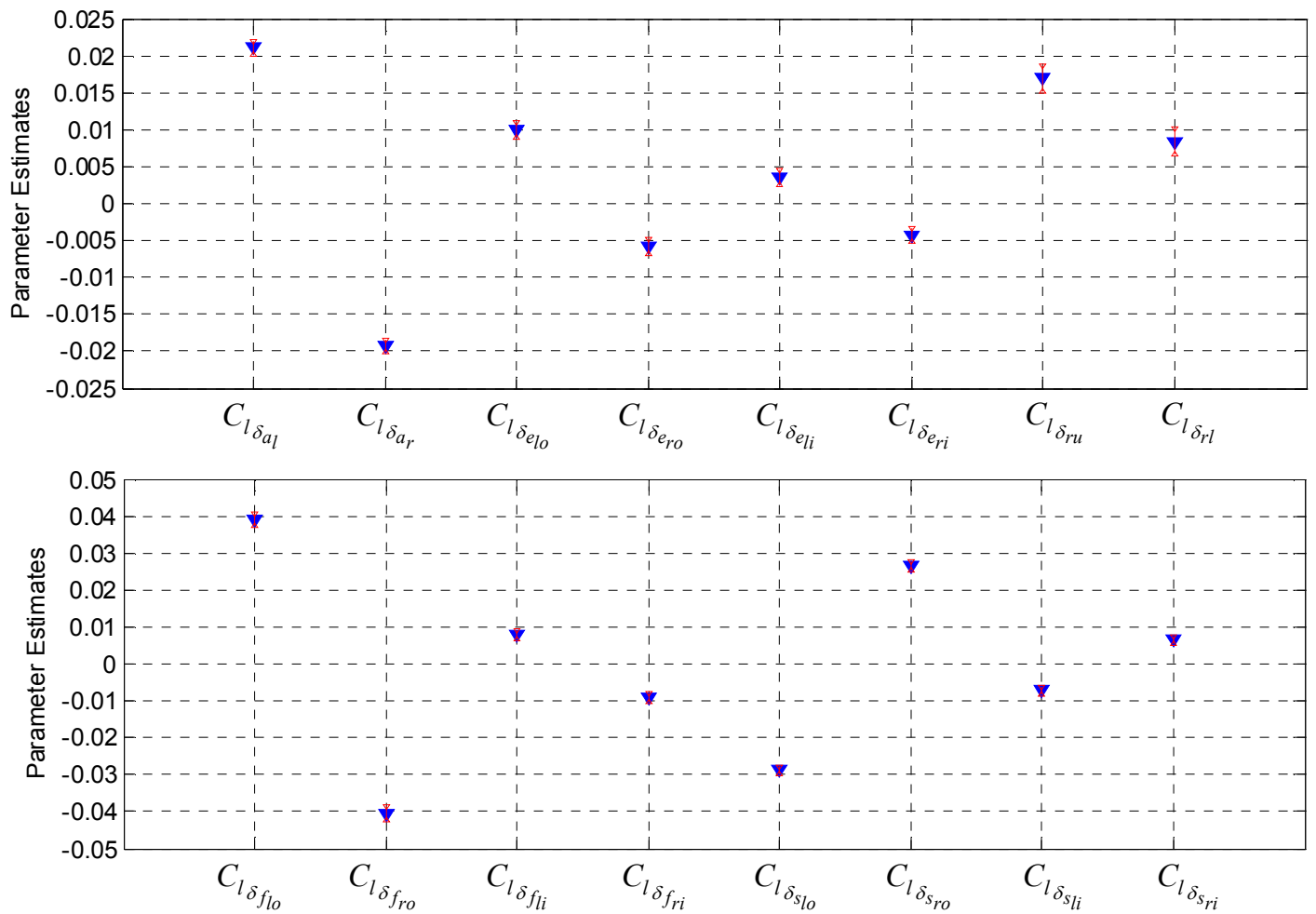

Figure 16. T-2 roll control effectiveness estimates from a single maneuver

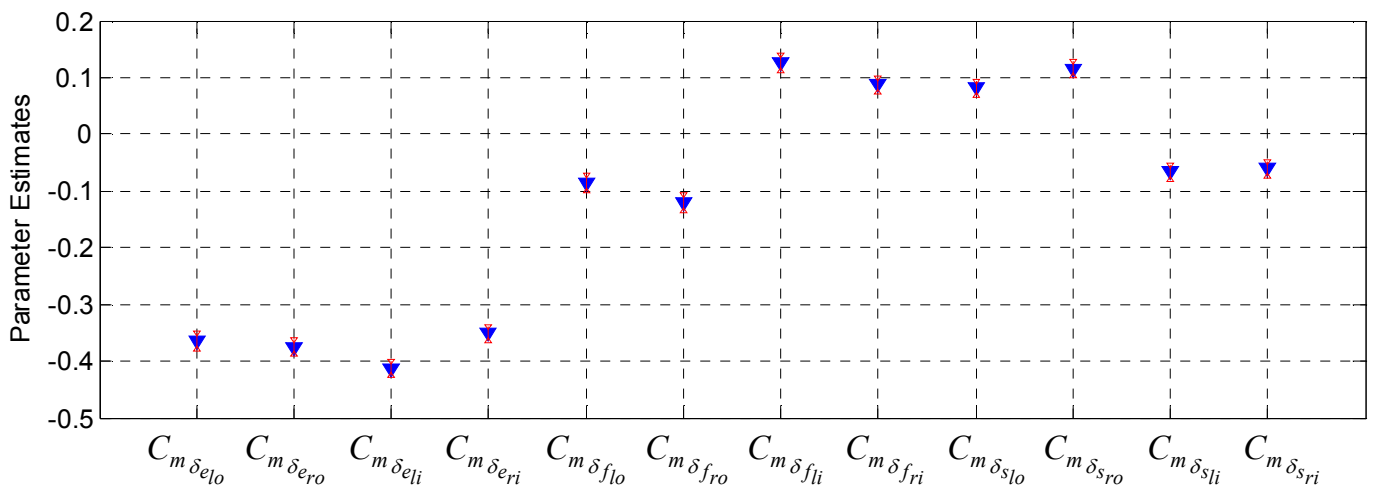

Figure 17. T-2 pitch control effectiveness estimates from a single maneuver

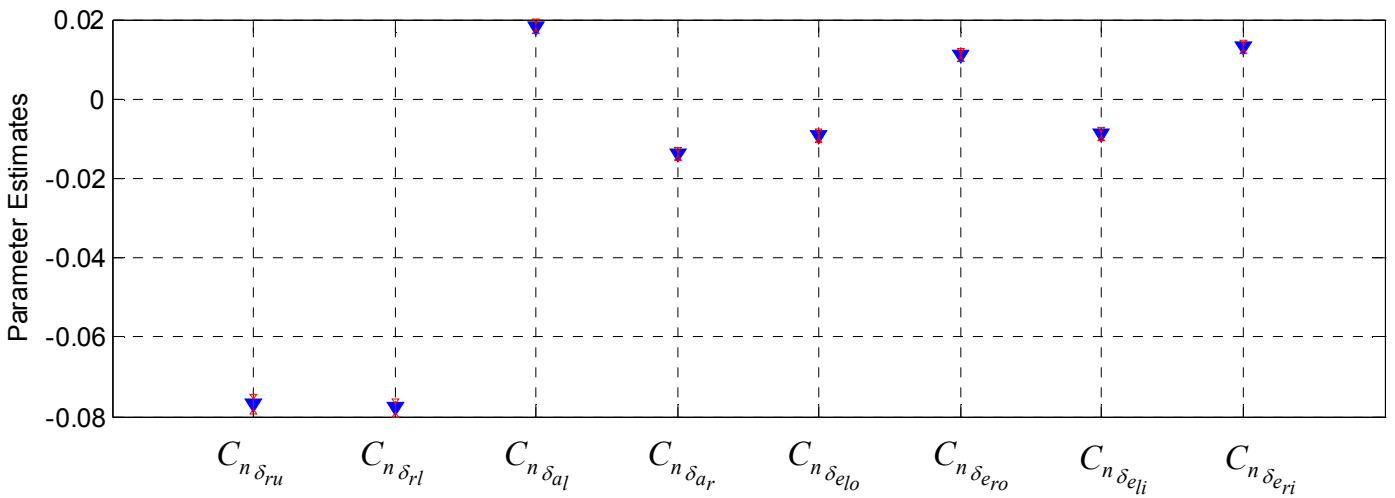

Figure 18. T-2 yaw control effectiveness estimates from a single maneuver 
Figures 16, 17, and 18 show control surface effectiveness per radian for roll, pitch, and yaw, respectively, estimated from a single 40 -sec flight test maneuver on the T-2 aircraft. Any control surface effectiveness missing from the figures (such as $C_{n_{\delta_{f_{l}}}}$ ) means the control effectiveness parameter estimate was statistically zero based on the flight data. This flight test maneuver provided data that allowed estimation of a large number of individual control surface effectiveness values with good accuracy in real time, including unconventional ones such as $C_{l_{f_{r i}}}$.

Because of test range boundaries, the $40 \mathrm{sec}$ maneuver included a level turn along with parts of two straight leg paths. This resulted in a change in nominal angle of attack from approximately 4 deg to 8 deg during the maneuver, but that did not affect control effectiveness estimates. From previous flight test experience, control surface effectiveness was expected to be linear and constant for low angles of attack and low control surface deflection angles, and this expectation was validated by the modeling results.

Individual control effectiveness estimates showed good physical consistency, with correct signs and reasonable magnitudes. Control surface effectiveness estimates for separate but similar surfaces on opposite sides exhibited equal effectiveness magnitudes but opposite signs, e.g. $C_{l_{\delta_{a_{l}}}}$ and $C_{l_{\delta_{a_{r}}}}$. Roll effectiveness of upper rudder was greater than for lower rudder, but the yaw effectiveness of these two control surfaces was approximately the same, as would be expected from geometry. The sum of individual surface effectiveness estimates matched well with corresponding estimates for those same surfaces moved together. For example, the sum of $C_{n_{\delta_{r_{u}}}}$ and $C_{n_{\delta_{\eta_{\eta}}}}$ estimates was approximately the same as the rudder yaw control effectiveness estimate $C_{n_{\delta_{r}}}$ when both rudder surfaces were moved together in the same way. Similar statements could be made about other control surfaces, such as the ailerons and elevators. The modeling results also provided information on relative effectiveness of individual control surfaces. For example, outboard flaps were found to be approximately twice as effective in roll as individual aileron surfaces, whereas the inboard flaps were only about half as effective in roll as individual aileron surfaces.

\section{Concluding Remarks}

Multi-axis optimal flight test input design has enabled new approaches to efficient stability and control flight testing that were previously not possible. Some of the flight test capabilities that can be realized with these new approaches were demonstrated using a dynamically-scaled jet transport aircraft. Flight test experiments included multi-axis stability and control flight testing at high nominal sideslip angles, in post-stall flight, and near stalls and departures; aerodynamic modeling over large portions of the flight envelope with a single maneuver, efficient flight envelope expansion, simulation validation, and simultaneously estimating control effectiveness for a large number of individual control surfaces. For all of these applications, use of an orthogonal optimized multi-axis input design technique in various ways resulted in highly efficient and practical flight testing with accurate modeling results.

The multiple simultaneous control surface movements necessary for orthogonal optimized multi-sine inputs cannot be implemented by a human pilot. Consequently, a requirement for using the flight test maneuvers described in this work is that the test aircraft must have an automated capability to add these excitation inputs to commands from the pilot and feedback control system, typically just before rate and position limiting is applied to the actuator commands. This flight testing approach allows the pilot to concentrate on attaining the desired flight condition or sequence of flight conditions, while the more difficult task of moving the control surfaces to excite the aircraft dynamics in all degrees of freedom over a range of frequencies can be done by the automated excitations. This procedure allows very efficient stability and control flight testing and highly accurate modeling results, with effective pilot oversight and low pilot workload.

Although the maneuvers described in this work were intended for stability and control flight testing, the same general approach can be applied more broadly for real-time modeling in changing flight conditions with various aircraft configurations, as well as real-time modeling for degraded aircraft, due to aging, damage, and failures. The fact that the inputs are optimized for minimum perturbation amplitudes and maximum input energy make them applicable to practical problems where exciting aircraft dynamic response excessively would disturb the pilot or passengers. Initial investigations of this approach have already begun, with the development of an onboard envelope protection system for a commuter aircraft in icing conditions ${ }^{7-9}$, and work studying the minimum necessary excitation for accurate stability and control parameter estimation in real time ${ }^{6}$. 


\section{Acknowledgments}

The efforts of the AirSTAR flight test team at NASA Langley, in building and testing the aircraft and associated systems, carefully calibrating the instrumentation, and carrying out the flight operations to collect the high-quality flight data used in this study, are gratefully acknowledged. Research in dynamic modeling is funded by the NASA Aviation Safety Program, Integrated Resilient Aircraft Control (IRAC) and Vehicle Safety Systems Technology (VSST) projects.

\section{References}

${ }^{1}$ Klein, V. and Morelli, E.A., Aircraft System Identification - Theory and Practice, AIAA Education Series, AIAA, Reston, VA, 2006.

${ }^{2}$ Morelli, E.A. "Multiple Input Design for Real-Time Parameter Estimation in the Frequency Domain," Paper REG-360, $13^{\text {th }}$ IFAC Symposium on System Identification, Rotterdam, The Netherlands, August 2003.

${ }^{4}$ Morelli, E.A., Derry, S.D., and Smith, M.S. "Aerodynamic Parameter Estimation of the X-43A (Hyper-X) from Flight Test Data," AIAA-2005-5921, AIAA Atmospheric Flight Mechanics Conference, San Francisco, CA, August 2005.

${ }^{5}$ Morelli, E.A. "Flight-Test Experiment Design for Characterizing Stability and Control of Hypersonic Vehicles," Journal of Guidance, Control, and Dynamics, Vol. 32, No. 3, May-June 2009, pp. 949-959.

${ }^{6}$ Morelli, E.A. and Smith, M.S. "Real-Time Dynamic Modeling - Data Information Requirements and Flight Test Results," Journal of Aircraft, Vol. 46, No. 6, November-December 2009, pp. 1894-1905.

${ }^{7}$ Gingras, D.R., Barnhart, B., Ranaudo, R., Ratvasky, T.P., and Morelli, E.A. "Envelope Protection for In-Flight Ice Contamination," AIAA-2009-1458, 47 $7^{\text {th }}$ AIAA Aerospace Sciences Meeting, Orlando, FL, January 2009.

${ }^{8}$ Ranaudo, R., Martos, B., Norton, B., Gingras, D.R., Barnhart, B. Ratvasky, T.P., and Morelli, E.A. "Piloted Simulation to Evaluate the Utility of a Real Time Envelope Protection System for Mitigating In-Flight Icing Hazards," AIAA-2010-7987, AIAA Atmosphere and Space Environments Conference, Toronto, Canada, August 2010.

${ }^{9}$ Gingras, D.R., Barnhart, B., Ranaudo, R., Martos, B., Ratvasky, T.P., and Morelli, E.A. "Development and Implementation of a Model-Driven Envelope Protection System for In-Flight Ice Contamination," AIAA-2010-8141, AIAA Guidance, Navigation, and Control Conference, Toronto, Canada, August 2010.

${ }^{10}$ Morelli, E.A. and Klein, V. "Application of System Identification to Aircraft at NASA Langley Research Center," Journal of Aircraft, Vol. 42, No. 1, January-February 2005, pp. 12-25.

${ }^{11} \mathrm{http}: / /$ dcb.larc.nasa.gov/SIDPAC/SIDBook_SIDPAC.htm

${ }^{12}$ Morelli, E.A., "Practical Aspects of the Equation-Error Method for Aircraft Parameter Estimation," AIAA-2006-6144, AIAA Atmospheric Flight Mechanics Conference, Keystone, CO, August 2006.

${ }^{13}$ Morelli, E.A. "Real-Time Parameter Estimation in the Frequency Domain," Journal of Guidance, Control, and Dynamics, Vol. 23, No. 5, September-October 2000, pp. 812-818.

${ }^{14}$ Press, W.H., S.A. Teukolsky, W.T. Vettering, and B.R. Flannery Numerical Recipes in FORTRAN: The Art of Scientific Computing, $2^{\text {nd }}$ Ed., Cambridge University Press, New York, NY, 1992, Chapter 10.

${ }^{15}$ Jordan, Thomas L., Langford, William M., and Hill, Jeffrey S.; "Airborne Subscale Transport Aircraft Research Testbed: Aircraft Model Development”, AIAA 2005-6432, AIAA Guidance, Navigation, and Control Conference and Exhibit, August 2005.

${ }^{16}$ Jordan, Thomas L., Foster, John V., Bailey, Roger M., and Belcastro, Christine M., "AirSTAR: A UAV Platform for Flight Dynamics and Control System Testing," AIAA-2006-3307, 25th AIAA Aerodynamic Measurement Technology and Ground Testing Conference, San Francisco, CA, June 2006.

${ }^{17}$ Murch, A. M., "A Flight Control System Architecture for the NASA AirSTAR Flight Test Facility," AIAA 2008-6990, AIAA Guidance, Navigation, and Control Conference and Exhibit, Honolulu, HI, August 2008.

${ }^{18}$ Cunningham, K., Foster, J.V., Morelli, E.A., and Murch, A.M., "Practical Application of a Subscale Transport Aircraft for Flight Research in Control Upset and Failure Conditions," AIAA-2008-6200, AIAA Atmospheric Flight Mechanics Conference, Honolulu, HI, August 2008.

${ }^{19}$ Shah, Gautam H., Cunningham, Kevin, Foster, John V., Fremaux, C. Michael, Stewart, Eric C., Wilborn, James E., Gato, William, Pratt, Derek W.; "Wind-Tunnel Investigation of Commercial Transport Aircraft Aerodynamics at Extreme Flight Conditions”, SAE 2002-01-2912, World Aviation Congress \& Display, November 2002. 
Table 1 Multiple input design for the T-2 subscale jet transport aircraft, level flight, $V_{o}=80 \mathrm{kts}, \alpha_{o}=5 \mathrm{deg}, T=10 \mathrm{sec}$

\begin{tabular}{|c|c|c|c|c|c|}
\hline Input & $A(\mathrm{deg})$ & $A_{k}(\mathrm{deg})$ & $k$ & $\phi_{k}(\mathrm{rad})$ & $R P F$ \\
\hline \multirow{7}{*}{$\delta_{e}$} & \multirow{7}{*}{2.0} & 0.3162 & 3 & 2.9478 & \multirow{7}{*}{1.03} \\
\hline & & 0.3873 & 6 & 0.6008 & \\
\hline & & 0.4472 & 9 & -2.6991 & \\
\hline & & 0.4472 & 12 & -1.6517 & \\
\hline & & 0.3873 & 15 & 2.6902 & \\
\hline & & 0.3162 & 18 & 2.0873 & \\
\hline & & 0.3162 & 21 & -2.8619 & \\
\hline \multirow{7}{*}{$\delta_{r}$} & \multirow{7}{*}{2.0} & 0.3162 & 2 & 2.8435 & \multirow{7}{*}{1.14} \\
\hline & & 0.3873 & 5 & 2.5259 & \\
\hline & & 0.4472 & 8 & 2.7562 & \\
\hline & & 0.4472 & 11 & -0.5132 & \\
\hline & & 0.3873 & 14 & -0.7433 & \\
\hline & & 0.3162 & 17 & 2.3959 & \\
\hline & & 0.3162 & 20 & -0.7581 & \\
\hline \multirow{7}{*}{$\delta_{a}$} & \multirow{7}{*}{1.0} & 0.3780 & 4 & 1.5438 & \multirow{7}{*}{1.15} \\
\hline & & 0.3780 & 7 & -1.6413 & \\
\hline & & 0.3780 & 10 & 1.2011 & \\
\hline & & 0.3780 & 13 & 1.0767 & \\
\hline & & 0.3780 & 16 & -2.3373 & \\
\hline & & 0.3780 & 19 & -2.3327 & \\
\hline & & 0.3780 & 22 & -2.7602 & \\
\hline
\end{tabular}

Table 2 T-2 Aircraft geometry and nominal mass properties

\begin{tabular}{|c|l|}
\hline $\bar{c}, \mathrm{ft}$ & 0.915 \\
\hline$b, \mathrm{ft}$ & 6.849 \\
\hline$S, \mathrm{ft}^{2}$ & 5.902 \\
\hline$x_{o}$, in & 57.30 \\
\hline$y_{o}$, in & 0.000 \\
\hline$z_{o}$, in & 11.28 \\
\hline$x_{c g}$, in & 56.63 \\
\hline$y_{c g}$, in & 0.000 \\
\hline$z_{c g}$, in & 11.43 \\
\hline$m$, slugs & 1.585 \\
\hline$I_{x}$, slugs- $\mathrm{ft}^{2}$ & 1.179 \\
\hline$I_{y}$, slugs- $\mathrm{ft}^{2}$ & 4.520 \\
\hline$I_{z}$, slugs- $\mathrm{ft}^{2}$ & 5.527 \\
\hline$I_{x z}$, slugs- $\mathrm{ft}^{2}$ & 0.211 \\
\hline
\end{tabular}


Table 3 T-2 maneuvers applying multi-axis excitation for slow approach to stall and recovery

\begin{tabular}{|c|c|c|c|}
\hline Configuration & Power Level & Flight & Card \\
\hline Cruise & IDLE & 5 & 10 \\
\hline Cruise & 40 percent & 6 & 11 \\
\hline Cruise & 50 percent & 6 & 12 \\
\hline Cruise & 60 percent & 6 & 13 \\
\hline Takeoff & IDLE & 6 & 15 \\
\hline Cruise & IDLE & 9 & 10 \\
\hline Powered Approach & IDLE & 9 & 17 \\
\hline Powered Approach & IDLE & 11 & 17 \\
\hline Cruise & IDLE & 34 & 17 \\
\hline Cruise & IDLE & 34 & 17 \\
\hline Cruise & IDLE & 36 & 17 \\
\hline Cruise & IDLE & 36 & 17 \\
\hline Cruise & IDLE & 37 & 17 \\
\hline Cruise & IDLE & 37 & 17 \\
\hline Cruise & IDLE & 38 & 17 \\
\hline Cruise & IDLE & 38 & 17 \\
\hline Cruise & IDLE & 38 & 17 \\
\hline Cruise & IDLE & 42 & 17 \\
\hline Cruise & IDLE & 42 & 17 \\
\hline Cruise & IDLE & 42 & 17 \\
\hline
\end{tabular}

